\title{
QUANTUM NETWORKS MODELLED BY GRAPHS
}

\author{
PAVEL EXNER AND OLAF POST
}

\begin{abstract}
Quantum networks are often modelled using Schrödinger operators on metric graphs. To give meaning to such models one has to know how to interpret the boundary conditions which match the wave functions at the graph vertices. In this article we give a survey, technically not too heavy, of several recent results which serve this purpose. Specifically, we consider approximations by means of "fat graphs" — in other words, suitable families of shrinking manifolds — and discuss convergence of the spectra and resonances in such a setting.
\end{abstract}

\section{INTRODUCTION}

Quantum mechanics on metric graphs is a subject with a long history which can be traced back to the paper of Ruedenberg and Scherr [21] on spectra of aromatic carbohydrate molecules elaborating an idea of L. Pauling. A new impetus came in the eighties from the need to describe semiconductor graph-type structures, cf. [10], and the interest to these problems driven both by mathematical curiosity and practical applications is steadily growing; we refer to [13, 14] or the proceedings [4] for a bibliography to the subject.

Since quantum graphs are supposed to model various real graph-like structures with the transverse size which is small but non-zero, one has to ask naturally how close are such system to an "ideal" graph in the limit of zero thickness. This problem is not easy and a reasonable complete answer is known in case of "fat graphs" with Neumann boundary conditions and similar systems. A pioneering work in this area was done by Freidlin and Wentzell [11] and the papers [15] and [20] can be mentioned as important milestones. We managed to contribute to this problem in a series of papers, [8], [19] and [9], in which we improved the approximation using the intrinsic geometry of the manifold only, demonstrating the norm resolvent convergence, and finally extending the approximation also to resonances by means of complex scaling.

While these results provide in our opinion a solid insight into the Neumann-type situation, we must acknowledge as the authors that the three papers are long and rather technical, and some may find them not easy to read. This motivated us to write the present survey in which we intend to describe this family of approximation results without switching in the heavy machinery; let the reader judge whether we have succeeded.

Before proceeding let us mention that there is an encouraging recent progress in the more difficult Dirichlet case, see $[2,12,17,7]$, however, we will not discuss it here.

Let us briefly describe the contents of the paper. In the next section we describe the two basic objects of this paper, quantum graphs and graph-like manifolds (cf. Figure 1). Section 3 is devoted to convergence of the discrete spectrum summarizing the main results of Ref. [8]. An extension to non-compact graphs and a resolvent convergence coming from [19] is given in Section 4. Finally, in Section 5 describe the results of Ref. [9] showing how the resonances on quantum graphs and graph-like manifolds approximate each other.

\section{QuANTUM NETWORKS AND GRAPHS}

2.1. Quantum graphs. Suppose that $X_{0}$ is a connected metric graph given by $(V, E, \partial, \ell)$ where $(V, E, \partial)$ is a usual graph, i.e., $V$ denotes the set of vertices, $E$ denotes the set of edges, $\partial: E \longrightarrow$ $V \times V$ associates to each edge $e$ the pair $\left(\partial_{-} e, \partial_{+} e\right)$ of its initial and terminal point (and therefore

Key words and phrases. metric graphs, Schrödinger operators, "fat graphs", Neumann boundary conditions, convergence of the spectra, resonances. 


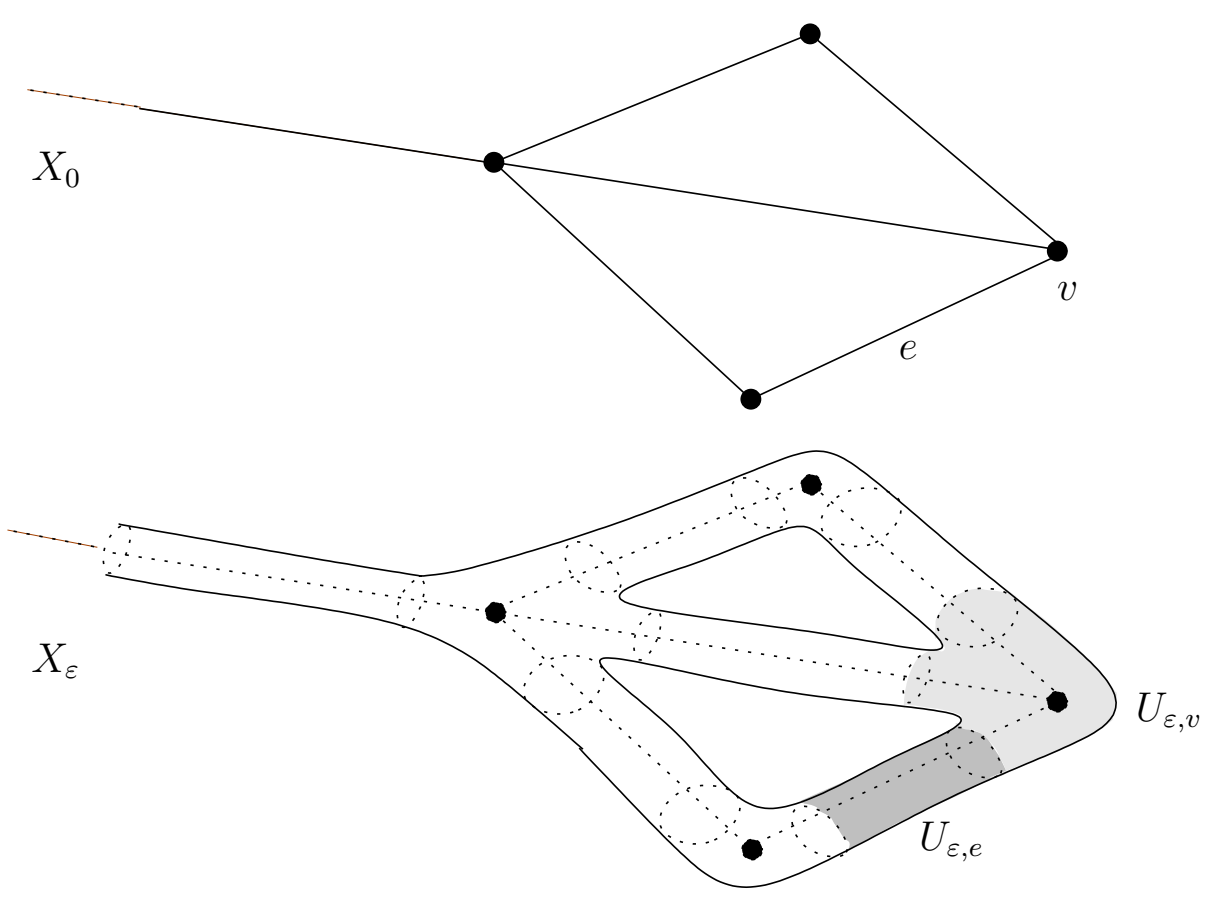

Figure 1. The metric graph $X_{0}$ with one external edge, five internal edges and four vertices and the associated graph-like manifold $X_{\varepsilon}$, here with cross section manifold $F=\mathbb{S}^{1}$.

an orientation). The space $X_{0}$ being a metric graph means that there is a length function $\ell: E \longrightarrow$ $(0, \infty]$ associating to each edge $e$ a length $\ell_{e}$. We often identify the edge $e$ with the interval $\left(0, \ell_{e}\right)$ and use $x=x_{e}$ as a coordinate. In addition, we denote $\mathrm{d} x=\mathrm{d} x_{e}$ the Lebesgue measure on $e$. In this way, $X_{0}$ becomes a topologically 1-dimensional space with singularities at the vertices. Moreover, $X_{0}$ carries a natural metric by defining the distance of two points to be the length of the shortest path in $\Gamma$ joining these points.

We call an edge e external iff $\ell_{e}=\infty$ and internal otherwise and we denote the corresponding sets by $E_{\text {ext }}$ and $E_{\text {int }}$. Properly speaking, the map $\partial$ is only defined on $E_{\text {int }} \times E_{\text {int }}$. For external edges, we do not consider the "end point" at infinity as a vertex of $\Gamma$, i.e., $\partial e$ contains only the initial vertex $\partial_{-} e=\partial e$. For each vertex $v \in V$ we set

$$
E_{v}^{ \pm}:=\left\{e \in E \mid \partial_{ \pm} e=v\right\} \quad \text { and } \quad E_{v}:=E_{v}^{+} \uplus E_{v}^{-},
$$

i.e., $E_{v}^{ \pm}$consists of all edges starting $(-)$resp. ending $(+)$at $v$ and $E_{v}$ is their disjoint union. Note that the disjoint union is necessary in order to allow single-edge loops, i.e., edges having the same initial and terminal point. We adopt the following uniform bounds on the degree $\operatorname{deg} v:=\left|E_{v}\right|$ and the length function $\ell$ :

$$
\begin{aligned}
1 \leq \operatorname{deg} v & \leq d_{0}, & & v \in V, \\
\ell_{e} & \geq \ell_{0}, & & e \in E,
\end{aligned}
$$

where $1 \leq d_{0}<\infty$ and $0<\ell_{0} \leq 1$. Needless to say, both the assumptions are fulfilled if $|E|$ and $|V|$ are finite.

We consider the Hilbert space defined naturally as the orthogonal sum,

$$
\mathcal{H}_{0}:=\mathrm{L}_{2}\left(X_{0}\right)=\bigoplus_{e \in E} \mathrm{~L}_{2}(e)
$$

A metric graph $X_{0}$ becomes a quantum graph when it is equipped with a self-adjoint operator $\Delta_{X_{0}}$ in the Hilbert space $\mathrm{L}_{2}\left(X_{0}\right)$. The latter is assumed to act as

$$
\left(\Delta_{X_{0}} f\right)_{e}:=-f_{e}^{\prime \prime}
$$


on each edge for $f \in \mathrm{H}^{2}\left(\Gamma^{1}\right)$, so a quantum graph is determined by fixing the vertex boundary conditions at each vertex, in order to turn the formal Laplacian into a self-adjoint operator. We will use the free (often called, not quite properly, Kirchhoff) boundary conditions: a function lies in the operator domain

$$
\mathcal{H}_{0}^{2}:=\operatorname{dom} \Delta_{X_{0}} \quad \text { iff } \quad f \in \bigoplus_{e \in E} \mathrm{H}^{2}(e)
$$

and the relations

$$
\begin{gathered}
f_{e_{1}}(v)=f_{e_{2}}(v), \quad e_{1}, e_{2} \in E_{v} \\
\sum_{e \in E_{v}} \overrightarrow{f_{e}^{\prime}}(v)=0
\end{gathered}
$$

are fulfilled for all $v \in V$ where

$$
\overrightarrow{f_{e}^{\prime}}(v):= \begin{cases}-f_{e}^{\prime}(0), & \text { if } v=\partial_{-} e \\ +f_{e}^{\prime}\left(\ell_{e}\right), & \text { if } v=\partial_{+} e\end{cases}
$$

defines the inward derivative of $f_{e}^{\prime}$ at $v$. Under the assumptions (2.1), the operator $\Delta_{X_{0}}$ is self-adjoint in $\mathcal{H}_{0}$ (cf. [14]). The quadratic form associated with the operator $\Delta_{X_{0}}$ is $\mathfrak{d}_{0}(f):=$ $\left\|\left(\Delta_{X_{0}}\right)^{1 / 2} f\right\|^{2}$ and can be expressed as

$$
\mathfrak{d}_{0}(f)=\left\|f^{\prime}\right\|^{2}=\sum_{e \in E}\left\|f_{e}^{\prime}\right\|^{2} \quad \text { on } \quad \mathcal{H}_{0}^{1}:=\mathrm{H}^{1}\left(X_{0}\right):=\mathrm{C}\left(X_{0}\right) \cap \bigoplus_{e \in E} \mathrm{H}^{1}(e) .
$$

2.2. Graph-like manifolds. Let us pass to a model of a quantum network which we will consider, corresponding to the idea that the graph has a small, but non-zero thickness. Let $X_{\varepsilon}$ be a $d$ dimensional connected manifold with metric $g_{\varepsilon}$ (cf. Figure 1). If $X_{\varepsilon}$ has boundary, we denote it by $\partial X_{\varepsilon}$; let us stress that our discussion covers different kind of models, "full" fat graphs considered by Kuchment and Zeng $[15,16]$ where the boundary is present, as well as "hollow" or "sleeve-type" manifolds having no boundary. We assume that $X_{\varepsilon}$ can be decomposed into open sets $U_{\varepsilon, e}$ and $U_{\varepsilon, v}$, i.e., ${ }^{1}$

$$
X_{\varepsilon}=\biguplus_{e \in E} U_{\varepsilon, e} \uplus \overline{\biguplus_{v \in V}} U_{\varepsilon, v} .
$$

Denote the metric on $X_{\varepsilon}$ by $g_{\varepsilon}$. We assume that $U_{\varepsilon, e}$ and $U_{\varepsilon, v}$ are isometric to

$$
\begin{array}{ll}
U_{\varepsilon, e} \cong\left(e \times F, g_{\varepsilon, e}\right) & g_{\varepsilon, e}=\mathrm{d} x_{e}^{2}+\varepsilon^{2} h \\
U_{\varepsilon, v} \cong\left(U_{v}, g_{\varepsilon, v}\right) & g_{\varepsilon, v}=\varepsilon^{2} g_{v}
\end{array}
$$

where $(F, h)$ is a compact $m$-dimensional manifold with $m:=(d-1)$, and $\left(U_{v}, g_{v}\right)$ is an $\varepsilon$ independent $d$-dimensional manifold ${ }^{2}$. Note that $\partial U_{\varepsilon, v} \backslash \partial X_{\varepsilon}$ has $(\operatorname{deg} v)$-many components isometric to $\left(F, \varepsilon^{2} h\right)$ denoted by $\left(\partial_{e} U_{v}, \varepsilon^{2} h\right)$ for $e \in E_{v}$. We sometimes write $U_{e}:=e \times F$.

Remark 1. For technical reasons (cf. Lemma 5) we assume that near $\partial_{e} U_{v}$, the (unscaled) manifold $\left(U_{v}, g_{v}\right)$ has a collar neighborhood $\left(\left(0, \ell_{0} / 2\right) \times F, \mathrm{~d} \hat{x}^{2}+\varepsilon^{2} h\right)$. If such a collar neighborhood happens to be too small, we can just change the decomposition (2.4) in such a way that we add a cylinder of length $\varepsilon \ell_{0} / 2$ (the length taken in the edge coordinates $x$ on $U_{e}$ ) from the edge neighborhood to the vertex neighborhood (becoming here a cylinder of length $\ell_{0} / 2$ in the vertex coordinates on $U_{v}$ since $\hat{x}=x / \varepsilon)$.

\footnotetext{
${ }^{1}$ Here and in the following, the expression $A=\biguplus_{i} A_{i}$ means that the $A_{i}$ 's are open (in $A$ ), mutually disjoint and the interior of $\bigcup_{i} \bar{A}_{i}$ equals $A$; recall that in an $\mathrm{L}_{2}$-theory it is enough to have charts covering a set of full measure.

${ }^{2}$ We employ just these particular charts; there is no need for a complete system. Strictly speaking, Figure 1 shows a slightly different situation where the edge neighborhoods are shortened (cf. Remark 2 below).
} 
The cross section manifold $F$ has a boundary or does not have one, depending on the analogous property of $X_{\varepsilon}$. For simplicity, we suppose that $\operatorname{vol}_{m} F=1$. Clearly, we have

$$
\mathrm{d} U_{\varepsilon, e}=\varepsilon^{m} \mathrm{~d} F \mathrm{~d} x_{e}
$$

for the Riemannian densities. We consider the Hilbert space

$$
\mathcal{H}_{\varepsilon}=\mathrm{L}_{2}\left(X_{\varepsilon}\right)
$$

and the Laplacian $\Delta_{X_{\varepsilon}} \geq 0$ (with Neumann boundary conditions if $\partial X_{\varepsilon} \neq \emptyset$ ) defined on

$$
\mathcal{H}_{\varepsilon}^{2}:=\operatorname{dom} \Delta_{X_{\varepsilon}}
$$

The associated quadratic form $\mathfrak{d}_{\varepsilon}(u):=\left\|\left(\Delta_{X_{\varepsilon}}\right)^{1 / 2} u\right\|^{2}$ can be expressed as

$$
\mathfrak{d}_{\varepsilon}(u)=\|\mathrm{d} u\|^{2} \quad \text { on } \quad \mathcal{H}_{\varepsilon}^{1}:=\mathrm{H}^{1}\left(X_{\varepsilon}\right)
$$

where $\mathrm{d} u$ is the exterior derivative of $u$. Note that the quadratic form expression contains only the metric $g_{\varepsilon}$, but no derivatives of $g_{\varepsilon}$.

Remark 2. We have chosen the full edge length on the edge neighborhood although this assumption is not valid if $X_{\varepsilon}$ is the $\varepsilon / 2$-neighborhood of a metric graph $X_{0}$ embedded into a Euclidean space from which it inherited its metric. In such a situation, however, the metric $g_{\varepsilon, e}$ differs from the metric $\mathrm{d} x_{e}^{2}+\varepsilon^{2} h$ only by a small longitudinal error. Using the fact that the Laplacian on $X_{\varepsilon}$ defined via its quadratic form depends only on $g_{\varepsilon}$ (and not on its derivatives), it can be shown that a small (uniform) perturbation of the product structure has only a small effect on the Laplacian, its spectrum, etc.

In addition, we assume that the following uniformity conditions are valid,

$$
c_{\mathrm{vol}}:=\sup _{v \in V} \operatorname{vol}_{d} U_{v}<\infty, \quad \lambda_{2}:=\inf _{v \in V} \lambda_{2}^{\mathrm{N}}\left(U_{v}\right)>0,
$$

where $\lambda_{2}^{\mathrm{N}}\left(U_{v}\right)$ denotes the second (i.e., first non-zero) Neumann eigenvalue of $\left(U_{v}, g_{v}\right)$.

Roughly speaking, the requirements (2.7) mean that the region $U_{v}$ remains small w.r.t. the vertex index — see the discussion in [19, Rem. 2.7]) for more details. Needless to say, these assumptions are trivially satisfied once the vertex set $V$ is finite.

Recall also one more domain related to the Laplacian for $\varepsilon \geq 0$. We denote by

$$
\mathcal{H}_{\varepsilon}^{k}:=\operatorname{dom}\left(\Delta_{X_{\varepsilon}}+1\right)^{k / 2}
$$

the scale of Hilbert spaces associated with the self-adjoint, non-negative operator $\Delta_{X_{\varepsilon}}$, together with its natural norm

For negative exponents, we set

$$
\|u\|_{k}:=\left\|\left(\Delta_{X_{\varepsilon}}+1\right)^{k / 2} u\right\|
$$

$$
\mathcal{H}_{\varepsilon}^{-k}:=\left(\mathcal{H}_{\varepsilon}^{k}\right)^{*}
$$

note that $\mathcal{H}_{\varepsilon}^{-k}$ can be again viewed as the completion of $\mathcal{H}_{\varepsilon} \subset \mathcal{H}_{\varepsilon}^{-k}$ with respect to the appropriate norm, in this case $\|u\|_{-k}=\left\|\left(\Delta_{X_{\varepsilon}}+1\right)^{-k / 2} u\right\|$.

\section{Convergence of Discrete spectrum}

Let us start with a simple thing, a convergence result using a variational characterization of discrete eigenvalues. We assume that $X_{0}$ is a compact metric graph, in other words,

$$
|E|<\infty \text { and } \ell_{e}<\infty \text { for all } e \in E .
$$

In this case, the graph-like manifold $X_{\varepsilon}$ is also compact, and the spectrum of $\Delta_{X_{\varepsilon}}, \varepsilon \geq 0$, is purely discrete. We denote by $\lambda_{k}(\varepsilon)$ the $k$-th eigenvalue of $\Delta_{X_{\varepsilon}}, k \geq 1$, repeated with respect to the multiplicity. The min-max variational characterization is then

$$
\lambda_{k}(\varepsilon)=\inf _{L_{k}} \sup _{u \in L_{k} \backslash\{0\}} \frac{\mathfrak{d}_{\varepsilon}(u)}{\|u\|^{2}}
$$


where the infimum is taken over all $k$-dimensional subspaces $L_{k}$ of $\mathrm{H}^{1}\left(X_{\varepsilon}\right)$.

Our main result in this section, coming from $[20,15,8]$, is the following:

Theorem 3. Assume that the metric graph $X_{0}$ is compact, i.e., the graph is finite and each edge has a finite length. Then the $k$-th (Neumann) eigenvalue of the Laplacian on the graph-like manifold $\lambda_{k}(\varepsilon)$ converges to the $k$-th eigenvalue of the Laplacian on the metric graph $X_{0}$ with the free boundary conditions. Specifically, we have

$$
\lambda_{k}(\varepsilon)-\lambda_{k}(0)=\mathcal{O}\left(\varepsilon^{1 / 2}\right)
$$

where the error term $\mathcal{O}\left(\varepsilon^{1 / 2}\right)$ depends on the index $k$.

The proof is based on an abstract comparison result. In order to compare the respective eigenvalues of the quantum graph and the graph-like manifold, we need identification maps expressed in terms of the quadratic forms, namely

$$
J^{1}: \mathcal{H}_{0}^{1} \longrightarrow \mathcal{H}_{\varepsilon}^{1} \quad \text { and } \quad J^{1 \prime}: \mathcal{H}_{\varepsilon}^{1} \longrightarrow \mathcal{H}_{0}^{1}
$$

We have the following abstract eigenvalue comparison result (see, e.g., [8, Lem. 2.1]):

Lemma 4. Assume that there are $\delta_{1}=\delta_{1}(\varepsilon)$ and $\delta_{2}=\delta_{2}(\varepsilon)$ such that

$$
\begin{array}{ll}
\mathfrak{d}_{0}(f)+\delta_{1}\|f\|_{1}^{2} \geq \mathfrak{d}_{\varepsilon}\left(J^{1} f\right), & \|f\|^{2}-\delta_{1}\|f\|_{1}^{2} \leq\left\|J^{1} f\right\|^{2}, \\
\mathfrak{d}_{\varepsilon}(u)+\delta_{2}\|u\|_{1}^{2} \geq \mathfrak{d}_{0}\left(J^{1 \prime} u\right), & \|u\|^{2}-\delta_{2}\|u\|_{1}^{2} \leq\left\|J^{1 \prime} u\right\|^{2}
\end{array}
$$

for all $f \in \mathcal{H}_{0}^{1}$ and $u \in \mathcal{H}_{\varepsilon}^{1}$. Then

$$
\frac{-2\left(1+\lambda_{k}(0)\right)\left(1+\delta_{1}\right)}{1-\left(\delta_{1}+\delta_{2}\left(1+\delta_{1}\right)\right)\left(1+\lambda_{k}(0)\right)} \delta_{2} \leq \lambda_{k}(\varepsilon)-\lambda_{k}(0) \leq \frac{2\left(1+\lambda_{k}(0)\right)}{1-\delta_{1}\left(1+\lambda_{k}(0)\right)} \delta_{1}
$$

where the upper bound is $\mathcal{O}\left(\delta_{1}\right)$ depending on $\lambda_{k}(0)$ and the lower bound is $\mathcal{O}\left(\delta_{2}\right)$ depending additionally on $\delta_{1}$.

In our concrete example, the $\mathcal{H}_{0}^{1} \rightarrow \mathcal{H}_{\varepsilon}^{1}$ identification operator can be chosen as

$$
J^{1} f(z):= \begin{cases}\varepsilon^{-m / 2} f_{e}(x) & \text { if } z=(x, y) \in U_{e}, \\ \varepsilon^{-m / 2} f(v) & \text { if } z \in U_{v}\end{cases}
$$

Note that the definition makes sense since functions in $\mathcal{H}_{0}^{1}$ are continuous. For the map in the opposite direction, we first introduce the following averaging operators

$$
\begin{gathered}
\left(N_{e} u\right)(x):=\left\langle\mathbb{1}, u_{e}(x, \cdot)\right\rangle_{F}=\int_{F} u_{e}(x, y) \mathrm{d} F(y), \\
C_{v} u:=\left\langle\mathbb{1}_{v}, u_{v}\right\rangle_{U_{v}}=\frac{1}{\operatorname{vol}_{d} U_{v}} \int_{U_{v}} u \mathrm{~d} U_{v}
\end{gathered}
$$

for $u \in \widetilde{\mathcal{H}}=\mathrm{L}_{2}\left(X_{\varepsilon}\right)$. What they yield is nothing else than the Fourier coefficient corresponding to the first (transverse) eigenfunction $\mathbb{1}(y)=1$ on $F$ and $\mathbb{1}_{v}(z)=\left(\operatorname{vol}_{d} U_{v}\right)^{-1}$ on $U_{v}$, respectively; note that these eigenfunctions are constant and that $\operatorname{vol}_{m} F=1$. We set

$$
\left(J^{1 \prime} u\right)_{e}(x):=\varepsilon^{m / 2}\left(N_{e} u(x)+\sum_{v \in \partial e} \rho(\operatorname{dist}(x, v))\left(C_{v} u-N_{e} u(v)\right)\right)
$$

for $x \in e$, where $\rho: \mathbb{R} \longrightarrow[0,1]$ is a smooth function with

$$
\rho(0)=1 \quad \text { and } \quad \rho(r)=0 \text { for all } r \geq \ell_{0} / 2 \text {. }
$$

The interpolating contribution related to $C_{v} u-N_{e} u(v)$ is needed in order to make $J^{1 \prime} u$ continuous at each vertex. The following lemma ensures that the error coming from this correction remains small: 
Lemma 5. We have

$$
\varepsilon^{m}\left|C_{v} u-N_{e} u(v)\right|^{2} \leq \frac{8}{\ell_{0}}\left(1+\frac{1}{\lambda_{2}}\right) \varepsilon\|\mathrm{d} u\|_{\varepsilon, v}^{2}
$$

for all $u \in \mathcal{H}_{\varepsilon}^{1}$ and $v \in \partial e$, where $\|\cdot\|_{\varepsilon, v}$ denotes the $\mathrm{L}_{2}$-norm on $U_{\varepsilon, v}$.

Proof. Due to Remark 1 , each component $\partial_{e} U_{v}$ of $\partial U_{v}$ has a collar neighborhood $\left(0, \ell_{0} / 2\right) \times F$ of length $\ell_{0} / 2$ (in the unscaled coordinates of $U_{v}$ ). The Cauchy-Schwarz inequality and the Sobolev trace estimate (see e.g. [14, Lem. 8])

$$
|f(0)|^{2} \leq \frac{8}{\ell_{0}} \int_{0}^{\ell_{0} / 2}\left(|f(s)|^{2}+\left|f^{\prime}(s)\right|^{2}\right) \mathrm{d} s
$$

yield (with $f(s)=f_{y}(s)=C_{v} u-u(s, y)$ in the collar coordinates, and then integration over $y \in F$ )

$$
\left|C_{v} u-N_{e} u(v)\right|^{2} \leq \int_{F}\left|C_{v} u-u(0, \cdot)\right|^{2} \mathrm{~d} F \leq \frac{8}{\ell_{0}}\left(\left\|C_{v} u-u\right\|_{v}^{2}+\|\mathrm{d} u\|_{v}^{2}\right),
$$

where $C_{v} u$ is considered to be a constant function on $U_{v}$ and $\|\cdot\|_{v}$ is the $\mathrm{L}_{2}$-norm on $U_{v}$. Now $C_{v} u-u$ is orthogonal to the first (constant) eigenfunction of the Neumann Laplacian on $U_{v}$, and the minmax principle ensures that the squared norm of $C_{v} u-u$ can be estimated by $\left(\lambda_{2}^{\mathrm{N}}\left(U_{v}\right)\right)^{-1}\|\mathrm{~d} u\|_{U_{v}}^{2}$. Using the scaling $g_{\varepsilon, v}=\varepsilon^{2} g_{v}$ of the metric and (2.7), we obtain the desired estimate.

We also have to make sure that eigenfunctions $u$ of $\Delta_{X_{\varepsilon}}$ belonging to eigenvalues bounded with respect to $\varepsilon$, cannot concentrate on the vertex neighborhoods:

Lemma 6. We have

$$
\|u\|_{\varepsilon, v}^{2} \leq c_{\mathrm{vx}} \varepsilon\left(\|\mathrm{d} u\|_{\varepsilon, v}^{2}+\|u\|_{\varepsilon, e}^{2}+\|\mathrm{d} u\|_{\varepsilon, e}^{2}\right)
$$

for $u \in \mathcal{H}_{\varepsilon}^{1}$, where $e$ is any edge adjacent to the vertex $v$. The constant $c_{\mathrm{vx}}$ in this inequality depends only on $\ell_{0}, \lambda_{2}$ and $c_{\mathrm{vol}}$.

Proof. We employ the estimate

$$
\|u\|_{\varepsilon, v} \leq\left\|u-C_{v} u\right\|_{\varepsilon, v}+\sqrt{\operatorname{vol}_{d} U_{\varepsilon, v}}\left(\left|C_{v} u-N_{e} u(v)\right|+\left|N_{e} u(v)\right|\right) .
$$

The first summand can be treated as in the previous proof, the second by Lemma 5 , and the last one by a Sobolev trace estimate on the edge neighborhood similar to (3.7), namely $\varepsilon^{m}\left|N_{e} u(v)\right|^{2} \leq$ $8\left(\|u\|_{\varepsilon, e}^{2}+\|\mathrm{d} u\|_{\varepsilon, e}^{2}\right) / \ell_{0}$.

Proof of Theorem 3. It remains to show that the conditions (3.3) are fulfilled. We do not give the details here referring to $[8$, Sec. 5]. The proof of (3.3a) is simple, and it works even with $\delta=0$, hence one obtains a stronger estimate, $\lambda_{k}(\varepsilon) \leq \lambda_{k}(0)$.

For the opposite inequality, we need to verify $(3.3 \mathrm{~b})$. To this end we need Lemma 5 in the norm and a quadratic form estimate. The norm estimate uses in addition Lemma 6 , and the estimate

$$
\|u\|_{\varepsilon, e}^{2}-\|N u\|_{\varepsilon, e}^{2} \leq \mathcal{O}(\varepsilon)\|u\|_{1}^{2}
$$

which follows from (4.2). For the quadratic form estimate we need the simple Cauchy-Schwarz bound $\|\mathrm{d} u\|_{\varepsilon, e}^{2} \leq\left\|(N u)^{\prime}\right\|_{\varepsilon, e}^{2}$.

Remark 7. Similar results can be obtained for more general situations when the vertex and edge neighborhoods scale at different rates, cf. [8, 16], and in certain situations also for the Dirichlet Laplacian, cf. [18], where, however, the resulting graph operator is decoupled. 


\section{Resolvent convergence}

Next we would like to go further and prove also results for non-compact graphs, and also convergence of eigenfunctions or resolvents. To do so we need some more notation. We write $\mathcal{H}_{\varepsilon}$ and $\Delta_{\varepsilon}=\Delta_{X_{\varepsilon}}$ for the $\varepsilon$-dependent spaces, $\varepsilon>0$. We stress that the parameter $\varepsilon$ enters only through the quantity $\delta=\delta_{\varepsilon}>0$ and one can interpret it as a label for the second Hilbert space involved - see also the appendix in [19] for the concept of a "distance" between two Hilbert spaces and associated non-negative operators. For brevity, we set

$$
R_{\varepsilon}:=\left(\Delta_{\varepsilon}+1\right)^{-1}, \quad \Delta_{\varepsilon}:=\Delta_{X_{\varepsilon}}, \quad \varepsilon \geq 0 .
$$

Definition 8. We say that an operator $J: \mathcal{H}_{0} \longrightarrow \mathcal{H}_{\varepsilon}$ is $\delta$-quasi-unitary with respect to $\Delta_{\varepsilon}$, iff $J^{*} J=\mathrm{id}_{0},\|J\|=1$, and

$$
\left.\left\|J J^{*}-\mathrm{id}_{\varepsilon}\right\|_{1 \rightarrow 0}=\|\left(J J^{*}-\mathrm{id}_{\varepsilon}\right) R_{\varepsilon}^{1 / 2}\right) \| \leq \delta,
$$

where $\operatorname{id}_{\varepsilon}$ is the identity on $\mathcal{H}_{\varepsilon}$, and $\|A\|_{1 \rightarrow 0}$ is the operator norm of $A: \mathcal{H}_{\varepsilon}^{1} \longrightarrow \mathcal{H}_{\varepsilon}^{0}$.

In our particular situation, we will employ the quasi-unitary operator

$$
J f:=\left\{f_{e} \otimes \mathbb{1}_{\varepsilon}\right\}_{e} \oplus\left\{0_{v}\right\}_{v},
$$

where $\mathbb{1}_{\varepsilon}=\varepsilon^{-m / 2} \mathbb{1}$ is the lowest normalized eigenfunction on $\left(F, \varepsilon^{2} h\right)$ and in turn $0_{v}$ is the zero function on $U_{v}$. The quasi-unitarity is stated in the following lemma:

Lemma 9. The map $J$ defined by (4.1) is $\delta_{\varepsilon}$-quasi-unitary, where $\delta_{\varepsilon}=\mathcal{O}\left(\varepsilon^{1 / 2}\right)$ depends only on $\ell_{0}, c_{\mathrm{vol}}$ and $\lambda_{2}$.

Proof. A simple calculation shows that $J^{*} J=\mathrm{id}_{0},\|J\|=1$, and that

$$
\left\|J J^{*} u-u\right\|^{2}=\sum_{e \in E}\left\|N_{e} u-u\right\|_{\varepsilon, e}^{2}+\sum_{v \in V}\|u\|_{\varepsilon, v}^{2} .
$$

The function $N_{e} u(x)-u(x, \cdot)$ is orthogonal to the constant function on $F$, and by the min-max principle we infer that

$$
\left\|N_{e} u-u\right\|_{\varepsilon, e}^{2} \leq \frac{\varepsilon^{2}}{\lambda_{2}(F)}\left\|\mathrm{d}_{F} u\right\|_{\varepsilon, e}^{2} \leq \frac{\varepsilon^{2}}{\lambda_{2}(F)}\|\mathrm{d} u\|_{\varepsilon, e}^{2}
$$

where $\lambda_{2}(F)$ is the first non-zero (Neumann) eigenvalue on $F$, and $\mathrm{d}_{F}$ is the derivative with respect to the transverse variable(s). The estimate of the sum over the vertex contributions follows from Lemma 6.

We also need a tool to compare the Laplacians on $\mathcal{H}_{0}$ and $\mathcal{H}_{\varepsilon}$. To this end we put:

Definition 10. We say that $\Delta_{\varepsilon}$ and $\Delta_{0}$ are $\delta$-close w.r.t. the map $J: \mathcal{H}_{0} \longrightarrow \mathcal{H}_{\varepsilon}$ iff

$$
\left\|J \Delta_{0}-\Delta_{\varepsilon} J\right\|_{2 \rightarrow-2}=\left\|R_{\varepsilon} J-J R_{0}\right\| \leq \delta
$$

where $\|A\|_{2 \rightarrow-2}$ denotes the operator norm of $A: \mathcal{H}_{0}^{2} \longrightarrow \mathcal{H}_{\varepsilon}^{-2}$.

Remark 11. Note that a 0-quasi-unitary map is indeed unitary. Furthermore, if $\Delta_{0}$ and $\Delta_{\varepsilon}$ are 0 -close with respect to a 0-quasi-unitary map $J$, then $\Delta_{0}$ and $\Delta_{\varepsilon}$ are unitarily equivalent. In this sense, the concept of quasi-unitarity and closeness provides a quantitative way to measure how far a pair of operators is from being unitarily equivalent.

In order to show that the operators $\Delta_{\varepsilon}$ and $\Delta_{0}$ are $\delta$-close, it is often easier to deal with the respective quadratic form domains as we have already done when demonstrating the convergence of the discrete spectrum. We thus want to compare the identification operators on the scale of order 1 with the quasi-unitary map $J$ :

Definition 12. We say that the identification maps (3.2) are $\delta$-compatible with the map $J: \mathcal{H}_{0} \longrightarrow$ $\mathcal{H}_{\varepsilon}$ iff

$$
\left\|J-J^{1}\right\|_{1 \rightarrow 0}=\left\|\left(J-J^{1}\right) R_{0}^{1 / 2}\right\| \leq \delta \quad \text { and } \quad\left\|J^{*}-J^{1 \prime}\right\|_{1 \rightarrow 0}=\left\|\left(J^{*}-J^{1 \prime}\right) R_{\varepsilon}^{1 / 2}\right\| \leq \delta .
$$


By means of an adjoint we obtain from $J^{1 \prime}$ a natural map

$$
J^{-1}:=\left(J^{1 \prime}\right)^{*}: \mathcal{H}_{0}^{-1} \longrightarrow \mathcal{H}_{\varepsilon}^{-1} .
$$

Now it is easy to derive the following criterion for $\delta$-closeness:

Lemma 13. Assume that $J^{1}$ and $J^{1 \prime}$ are $\delta$-compatible w.r.t. the map $J$, and that

$$
\left\|J^{-1} \Delta_{0}-\Delta_{\varepsilon} J^{1}\right\|_{1 \rightarrow-1}=\left\|R_{\varepsilon}^{1 / 2}\left(J^{-1} \Delta_{0}-\Delta_{\varepsilon} J^{1}\right) R_{0}^{1 / 2}\right\| \leq \delta .
$$

Then $\Delta_{\varepsilon}$ and $\Delta_{0}$ are $3 \delta$-close with respect to $J$.

We first check that the identification maps $J^{1}$ and $J^{1 \prime}$ are indeed $\mathcal{O}\left(\varepsilon^{1 / 2}\right)$-compatible with respect to the map $J$ :

Lemma 14. The maps $J^{1}$ and $J^{1 \prime}$ as defined in (3.4) and (3.5) are $\delta_{\varepsilon}$-compatible with $J$, where $\delta_{\varepsilon}=\mathcal{O}\left(\varepsilon^{1 / 2}\right)$ depends only on $\ell_{0}, d_{0}, c_{\mathrm{vol}}$ and $\lambda_{2}$.

Proof. We have

$$
\left\|\left(J-J^{1}\right) f\right\|^{2}=\varepsilon^{-m} \sum_{v \in V}\left(\operatorname{vol}_{d} U_{\varepsilon, v}\right)|f(v)|^{2}=\varepsilon \sum_{v \in V}\left(\operatorname{vol}_{d} U_{v}\right)|f(v)|^{2} .
$$

By a standard Sobolev estimate - see, e.g., estimate (3.7) or [19, Lem. 2.4]) - we can estimate the latter sum by $8 \varepsilon c_{\mathrm{vol}}\|f\|_{1}^{2} / \ell_{0}$. For the other identification operator we have

$$
\left\|\left(J^{*}-J^{1 \prime}\right) u\right\|^{2}=\sum_{e \in E} \sum_{v \in \partial e} \int_{0}^{\ell_{0} / 2} \rho(r)^{2} \varepsilon^{m}\left|C_{v} u-N_{e} u(v)\right|^{2} \mathrm{~d} r .
$$

Using now Lemma 5 and reordering the sum, we obtain the additional factor $d_{0}$, the maximum degree of a vertex, and the second estimate follows as well.

Next, we will now indicate briefly how to prove the closeness of the Laplacians:

Lemma 15. The Laplacians $\Delta_{\varepsilon}$ and $\Delta_{0}$ are $\delta_{\varepsilon}$-close with respect to the map $J$ defined in (4.1) where $\delta_{\varepsilon}=\mathcal{O}\left(\varepsilon^{1 / 2}\right)$ depends only on $\ell_{0}, d_{0}$ and $\lambda_{2}$.

Proof. We check the condition (4.3) of Lemma 13 which reduces to estimating

$$
\left|\mathfrak{d}_{0}\left(f, J^{1 \prime} u\right)-\mathfrak{d}_{\varepsilon}\left(J^{1} f, u\right)\right| \leq \sum_{e \in E} \sum_{v \in \partial e}\left\|f^{\prime}\right\|_{e} \int_{0}^{\ell_{0} / 2}\left|\rho^{\prime}(r)\right|^{2} \mathrm{~d} r \varepsilon^{m / 2}\left|C_{v} u-N_{e} u(v)\right| .
$$

in terms of $\delta_{\varepsilon}\|f\|_{1}\|u\|_{1}$; the claim follows from Lemma 5 and Cauchy-Schwarz.

Putting together the previous results, we come to the following conclusion:

Theorem 16. Adopt the uniformity conditions (2.1) and (2.7). Then the Laplacians $\Delta_{X_{\varepsilon}}$ and $\Delta_{X_{0}}$ are $\mathcal{O}\left(\varepsilon^{1 / 2}\right)$-close with respect to the quasi-unitary map $J$ defined in (4.1), i.e.

$$
\left\|\left(\Delta_{X_{\varepsilon}}+1\right)^{-1} J-J\left(\Delta_{X_{0}}+1\right)^{-1}\right\| \leq \mathcal{O}\left(\varepsilon^{1 / 2}\right),
$$

where the error term depends only on $\ell_{0}, d_{0}, c_{\mathrm{vol}}$ and $\lambda_{2}$. In addition, we have

$$
\left\|\left(\Delta_{X_{\varepsilon}}+1\right)^{-1}-J\left(\Delta_{X_{0}}+1\right)^{-1} J^{*}\right\| \leq \mathcal{O}\left(\varepsilon^{1 / 2}\right) .
$$

Proof. The first estimate follows from Lemmata 13-15; the second one in turn is a consequence of the first estimate and Lemma 9.

One can now develop the standard functional calculus for the Laplacians $\Delta_{\varepsilon}$ and $\Delta_{0}$, and deduce estimates similar to the ones in Theorem 16, but with the resolvent replaced by more general functions $\varphi\left(\Delta_{\varepsilon}\right)$ of the Laplacians. Specifically, $\varphi$ need to be measurable, continuous in a neighborhood of the spectrum of $\Delta_{0}$, and the limit at infinity must exist. For example, one can control the heat operators via $\varphi_{t}(\lambda)=\mathrm{e}^{-t \lambda}$ or the spectral projectors via $\varphi=\mathbb{1}_{I}$. A proof of the following result can be found in the appendices of the paper [19], see also [9]: 
Theorem 17. Under the assumptions of the previous theorem, we have

$$
\left\|\mathbb{1}_{I}\left(\Delta_{X_{\varepsilon}}\right) J-J \mathbb{1}_{I}\left(\Delta_{X_{0}}\right)\right\| \leq \mathcal{O}\left(\varepsilon^{1 / 2}\right) \quad \text { and } \quad\left\|\mathbb{1}_{I}\left(\Delta_{X_{\varepsilon}}\right)-J \mathbb{1}_{I}\left(\Delta_{X_{0}}\right) J^{*}\right\| \leq \mathcal{O}\left(\varepsilon^{1 / 2}\right)
$$

for the spectral projections provided $I$ is a compact interval such that $\partial I \cap \sigma\left(\Delta_{X_{0}}\right)=\emptyset$. In particular, if I contains a single eigenvalue $\lambda(0)$ of $\Delta_{X_{0}}$ with multiplicity one corresponding to an eigenfunction $u(0)$, then there is an eigenvalue $\lambda(\varepsilon)$ and an eigenfunction $u(\varepsilon)$ of $\Delta_{X_{\varepsilon}}$ such that

$$
\|J u(0)-u(\varepsilon)\|=\mathcal{O}\left(\varepsilon^{1 / 2}\right) .
$$

In addition, the spectra converge uniformly on $[0, \Lambda]$, i.e.

$$
\sigma\left(\Delta_{X_{\varepsilon}}\right) \cap[0, \Lambda] \rightarrow \sigma\left(\Delta_{X_{0}}\right) \cap[0, \Lambda]
$$

in the sense of Hausdorff distance on compact subsets of $[0, \Lambda]$. The same result is true if we consider only the essential or the discrete spectral components.

Naturally, the above stated spectral convergence reduces to the claim of Theorem 3 in the situation when the spectra are purely discrete.

\section{Convergence of Resonances}

In the final section we will deal with the convergence of resonances in the present setting. It is useful to include into the considerations also eigenvalues embedded in the continuous spectrum, because it may happen that resonances of a "fat graph" converge to such an eigenvalue, as it can be seen, e.g., in a simple motivating example of the metric graph consisting of a single loop with a half-line "lead" attached [9].

A standard and successful method of dealing with resonances is based on the concept of complex scaling, often an exterior one. The method has its roots in the seminal papers [1,3] and a lot of work was devoted to it; we refer to [9] for a sample bibliography. The main virtue is that it allows to reformulate treatment of resonances, i.e. poles of the analytically continued resolvent, and embedded eigenvalues, to analysis of discrete eigenvalues of a suitable non-selfadjoint operator. As we will see below the complex-scaling approach suits perfectly, in particular, to our convergence analysis.

In this section, we assume that the metric graph is finite, but non-compact, i.e.

$$
\left|E_{\text {int }}\right|<\infty \quad \text { and } \quad 0<\left|E_{\text {ext }}\right|<\infty,
$$

which means, in particular, that the assumptions (2.1) and (2.7) are satisfied.

5.1. Exterior scaling. We decompose the metric graph $X_{0}$ and the graph-like manifold $X_{\varepsilon}$ into an interior and exterior part $X_{\varepsilon, \text { int }}$ and $X_{\varepsilon \text {,ext }}$, respectively. For technical reasons, it is easier to do the cut not at the initial vertices $\partial e$ of an external edge $e \in E_{\text {ext }}$, but at a fixed distance, say one, from $\partial e$ along $e$, and similarly for the graph-like manifold. We therefore consider the internal metric graph $X_{0, \text { int }}$ consisting of all vertices, all edges of finite length and the edge parts $(0,1)$ for each external edge. The exterior metric graph $X_{0 \text {,ext }}$ is just the disjoint union of $\left|E_{\text {ext }}\right|$-many copies of a half-line $[0, \infty)$, and we use the corresponding parametrization on an external edge. In other words, we do not regard the boundary points $\Gamma_{0}=\partial X_{0 \text {,int }} \cap \partial X_{0 \text {,ext }}$ as vertices. Similarly, let $\Gamma_{\varepsilon}$ be the common boundary of $X_{\varepsilon, \text { int }}$ and $X_{\varepsilon \text {,ext }}$; note that $\Gamma_{\varepsilon}$ is isometric to $\left|E_{\text {ext }}\right|$-many copies of $\left(F, \varepsilon^{2} h\right)$.

Now we introduce the exterior dilation operator. For $\theta \in \mathbb{R}$ we define by

$$
\begin{aligned}
& \left(U_{0}^{\theta} f\right)(x):=\left\{\begin{array}{ll}
f(x), & x \in X_{0, \text { int }} \\
\mathrm{e}^{\theta / 2} f\left(\mathrm{e}^{\theta} x\right), & x \in X_{0, \mathrm{ext}}
\end{array}\right. \text { and } \\
& \left(U_{\varepsilon}^{\theta} u\right)(z):= \begin{cases}u(z), & z \in X_{\varepsilon, \text { int }} \\
\mathrm{e}^{\theta / 2} u\left(\mathrm{e}^{\theta} x, y\right), & z=(x, y) \in X_{\varepsilon, \mathrm{ext}}\end{cases}
\end{aligned}
$$


one-parameter unitary groups on $\mathcal{H}_{0}=\mathrm{L}_{2}\left(X_{0}\right)$ and $\mathcal{H}_{\varepsilon}=\mathrm{L}_{2}\left(X_{\varepsilon}\right)$, respectively, acting non-trivially on the external part only. We call the operator

$$
H_{\varepsilon}^{\theta}:=U_{\varepsilon}^{\theta} \Delta_{X_{\varepsilon}} U_{\varepsilon}^{-\theta}
$$

for $\varepsilon \geq 0$ the dilated Laplacian on $X_{\varepsilon}$ with the domain $\operatorname{dom} H_{\varepsilon}^{\theta}:=U_{\varepsilon}^{\theta}\left(\mathcal{H}_{\varepsilon}^{2}\right)$ for real $\theta$. A simple calculation shows that $\left(H_{\varepsilon}^{\theta} u\right)_{e}=\left(\Delta_{X_{\varepsilon}} u\right)_{e}$ holds for internal edges and that

$$
\left(H_{0}^{\theta} f\right)_{e}=-\mathrm{e}^{-2 \theta} f_{e}^{\prime \prime} \quad \text { and } \quad\left(H_{\varepsilon}^{\theta} u\right)_{e}=-\mathrm{e}^{-2 \theta} \partial_{x x} u_{e}+\frac{1}{\varepsilon^{2}} \Delta_{F} u_{e}
$$

is true for external edges with the domain $\mathcal{H}_{\varepsilon}^{2, \theta}:=\operatorname{dom} H_{\varepsilon}^{\theta}$ given by

$$
\mathcal{H}_{\varepsilon}^{2, \theta}:=\left\{f \in \mathrm{H}^{2}\left(X_{\varepsilon, \text { int }}\right) \oplus \mathrm{H}^{2}\left(X_{\varepsilon, \text { ext }}\right) \mid u_{\text {ext }}=\mathrm{e}^{\theta / 2} u_{\text {int }}, \quad \vec{u}_{\text {ext }}^{\prime}=\mathrm{e}^{3 \theta / 2} \vec{u}_{\text {int }}^{\prime} \text { on } \Gamma_{\varepsilon}\right\} .
$$

Here, $\mathrm{H}^{2}\left(X_{\varepsilon, \bullet}\right)$ are the functions from $\operatorname{dom} \Delta_{X_{\varepsilon}}$ restricted to $X_{\varepsilon, \bullet}$, i.e. without any condition at $\Gamma_{\varepsilon}$. In addition, $\vec{u}_{\bullet}^{\prime}$ is defined as the longitudinal derivative on the common boundary $\Gamma_{\varepsilon}$ oriented away from the internal part.

The expression of $H_{\varepsilon}^{\theta}$ now can be generalized to complex $\theta$ in the strip

$$
S_{\vartheta}=\{\theta \in \mathbb{C}|| \operatorname{Im} \theta \mid<\vartheta / 2\}
$$

where $0 \leq \vartheta<\pi$; we call the corresponding $H_{\varepsilon}^{\theta}$ the complex dilated Laplacian. The operators $\left\{H_{\varepsilon}^{\theta}\right\}_{\theta}$ form a family with spectrum contained in the common sector

$$
\Sigma_{\vartheta}:=\{z \in \mathbb{C}|| \arg z \mid \leq \vartheta\} .
$$

Moreover, we can determine the essential spectrum coming from the external part. Note that the branches of the essential spectrum associated with the higher transverse eigenvalues $\lambda_{k}(F), k \geq 2$, on the graph-like manifold all vanish as $\varepsilon \rightarrow 0$.

Lemma 18. Let the metric graph be finite and non-compact, i.e. (5.1) is fulfilled. Then

$$
\sigma_{\text {ess }}\left(H_{0}^{\theta}\right)=\mathrm{e}^{-2 \theta}[0, \infty) \quad \text { and } \quad \sigma_{\text {ess }}\left(H_{\varepsilon}^{\theta}\right)=\frac{1}{\varepsilon^{2}} \bigcup_{k \in \mathbb{N}}\left(\lambda_{k}(F)+\mathrm{e}^{-2 \theta}[0, \infty)\right) \text {. }
$$

In particular, since $\lambda_{1}(F)=0$, we have

$$
\sigma_{\mathrm{ess}}\left(H_{\varepsilon}^{\theta}\right) \cap B=\mathrm{e}^{-2 \theta}[0, \infty) \cap B
$$

for any bounded set $B \subset \mathbb{C}$ provided $\varepsilon>0$ is small enough.

In addition, we have the following important result:

Theorem 19. For $z$ not contained in the $\vartheta$-sector $\Sigma_{\vartheta}$, the resolvents

$$
R_{\varepsilon}^{\theta}(z):=\left(H_{\varepsilon}^{\theta}-z\right)^{-1}
$$

depend analytically on $\theta \in S_{\vartheta}$.

This is a highly non-trivial fact since $H_{\varepsilon}^{\theta}$ is neither of type A nor of type $\mathrm{B}$, in other words, both the sesquilinear form and the operator domains depend on $\theta$ even for real $\theta$. To put it differently, the (non-smooth) exterior scaling as defined here is a very singular perturbation of the operator $\Delta_{X_{\varepsilon}}=H_{\varepsilon}^{0}$. The main idea is to compare $R_{\varepsilon}^{\theta}$ with the resolvent of a decoupled operator, where one imposes Dirichlet boundary conditions at $\Gamma_{\varepsilon}$. An inspiration for such an idea was used in [5]; for a full proof in our situation we refer to [9]. As a consequence, we have the following result on the discrete spectrum. ${ }^{3}$

Lemma 20. The discrete spectrum of $H_{\varepsilon}^{\theta}$ is locally constant in $\theta$. As a consequence, discrete (complex) eigenvalues are "revealed" if $\operatorname{Im} \theta$ is positive and large enough. The same is true for eigenvalues embedded into the continuous spectrum $[0, \infty)$ of the Laplacian; in this case it is sufficient to have $\operatorname{Im} \theta>0$.

\footnotetext{
${ }^{3}$ One can derive also other spectral properties, e.g., $\sigma_{\mathrm{sc}}\left(H_{\varepsilon}^{\theta}\right)=\emptyset$ - see [9, Prop. 5.8] for more details.
} 
This is crucial for the above mentioned reformulation. Recall that by the most common definition a resonance is a pole in a meromorphic continuation of the resolvent over the cut corresponding to the essential spectrum into the "unphysical sheet" of the Riemann energy surface [6]. Rotating the essential spectrum one can reveal these singularities; this allows us to identify a resonance of $H_{\varepsilon}^{0}=\Delta_{X_{\varepsilon}}$ with a complex $\mathrm{L}_{2}$-eigenvalue of the dilated operator $H_{\varepsilon}^{\theta}$ for $\operatorname{Im} \theta$ large enough. Notice also that such a definition is consistent: it does not depend on where we cut the spaces into an interior and exterior part; of course, as far as the interior part remains compact.

5.2. Convergence result. In order to demonstrate convergence properties of resonances, we need to introduce a scale of Hilbert spaces associated to the non-selfadjoint operator $H_{\varepsilon}^{\theta}$. In particular, we set $\mathcal{H}^{2, \theta}:=\operatorname{dom} H_{\varepsilon}^{\theta}$ with the norm $\|u\|_{2, \theta}:=\left\|\left(H_{\varepsilon}^{\theta}+1\right) u\right\|$ and $\mathcal{H}^{-2, \theta}:=\left(H_{\varepsilon}^{\bar{\theta}}\right)^{*}$ with the dual norm; for details we refer to [9, App. A].

Definition 21. We say that an operator $J: \mathcal{H}_{0} \longrightarrow \mathcal{H}_{\varepsilon}$ is $\delta$-quasi-unitary with respect to $H_{\varepsilon}^{\theta}$, iff $J^{*} J=\mathrm{id}_{0},\|J\|=1$, and

$$
\left.\left\|J J^{*}-\mathrm{id}_{\varepsilon}\right\|_{2, \theta \rightarrow 0}=\|\left(J J^{*}-\mathrm{id}_{\varepsilon}\right) R_{\varepsilon}^{\theta}\right) \| \leq \delta,
$$

where $R_{\varepsilon}^{\theta}:=\left(H_{\varepsilon}^{\theta}+1\right)^{-1}$ and $\|A\|_{2, \theta \rightarrow 0}$ is the operator norm of $A: \mathcal{H}_{\varepsilon}^{2, \theta} \longrightarrow \mathcal{H}_{\varepsilon}^{0}$.

It is much easier to use the $\delta$-quasi-unitarity with respect to the non-dilated operator (via its quadratic form) as in Definition 8. In particular, we would like to compare the non-dilated scale $\mathcal{H}_{\varepsilon}^{1}$ of order 1 and the dilated scale $\mathcal{H}_{\varepsilon}^{2, \theta}$ of order 2 :

Definition 22. $\Delta_{\varepsilon}$ and $H_{\varepsilon}^{\theta}$ are compatible if there is a family of bounded, invertible operators $T_{\varepsilon}^{\theta}$ on $\mathcal{H}_{\varepsilon}$ such that $T_{\varepsilon}^{-\theta}=\left(T_{\varepsilon}^{\theta}\right)^{-1}, T_{\varepsilon}^{\bar{\theta}}=\left(T_{\varepsilon}^{\theta}\right)^{*}$ and $T_{\varepsilon}^{-\theta}\left(\mathcal{H}_{\varepsilon}^{1}\right) \subset \mathcal{H}_{\varepsilon}^{2, \theta}$. Given $\varepsilon \geq 0$, we say that $\Delta_{\varepsilon}$ and $H_{\varepsilon}^{\theta}$ are uniformly compatible with respect to $\varepsilon$, if there is a constant $C^{\theta}$, independent of $\varepsilon$, such that

$$
\left\|T_{\varepsilon}^{\theta}\right\|_{2, \theta \rightarrow 1}=\left\|\left(\Delta_{\varepsilon}+1\right)^{1 / 2} T_{\varepsilon}^{\theta} R_{\varepsilon}^{\theta}\right\| \leq C^{\theta} \quad \text { and } \quad\left\|R_{\varepsilon}^{\theta}\right\| \leq C^{\theta} .
$$

In the situation we consider there is a natural candidate for $T_{\varepsilon}^{\theta}$, namely

$$
T_{\varepsilon}^{\theta} u:=u_{\text {int }} \oplus \mathrm{e}^{\theta / 2} u_{\text {ext }} .
$$

In contrast to $U_{\varepsilon}^{\theta}$, the operators $T_{\varepsilon}^{\theta}$ are defined also for complex values of $\theta$. Again, as for the analyticity, the proof of uniform compatibility in our example needs some technical preliminaries which we skip here. In essence, one needs to define the resolvent $R_{\varepsilon}^{\theta}$ as a bounded operator from $\mathcal{H}^{-1, \theta}$ to $\mathcal{H}^{1, \theta}$ with $\varepsilon$-independent bound, where

$$
\mathcal{H}^{1, \theta}:=T^{\theta}\left(\mathcal{H}^{1}\right)=\left\{f \in \mathrm{H}^{1}\left(X_{\varepsilon, \text { int }}\right) \oplus \mathrm{H}^{1}\left(X_{\varepsilon, \text { ext }}\right) \mid u_{\text {ext }}=\mathrm{e}^{\theta / 2} u_{\text {int }} \text { on } \Gamma_{\varepsilon}\right\}
$$

is an appropriate space of order 1 for $H_{\varepsilon}^{\theta}$, not necessarily related to $\operatorname{dom}\left(H_{\varepsilon}^{\theta}+1\right)^{1 / 2}$.

Lemma 23. For a given $\varepsilon>0$, the operators $\Delta_{X_{\varepsilon}}$ and its complex dilated counterparts $H_{\varepsilon}^{\theta}$ are (uniformly) compatible. In particular, the map $J$ as defined in (4.1) is $\mathcal{O}\left(\varepsilon^{1 / 2}\right)$-quasi-unitary with respect to $H_{\varepsilon}^{\theta}$, where $\mathcal{O}\left(\varepsilon^{1 / 2}\right)$ depends on $\theta$.

Proof. The compatibility is demonstrated in [9, App. C]. The second assertion follows from

$$
\begin{aligned}
\left.\|\left(J J^{*}-\mathrm{id}_{\varepsilon}\right) R_{\varepsilon}^{\theta}\right) \| & \left.\leq\left\|T_{\varepsilon}^{-\theta}\right\| \cdot \|\left(J J^{*}-\mathrm{id}_{\varepsilon}\right)\left(\Delta_{\varepsilon}+1\right)^{-1 / 2}\right)\|\cdot\|\left(\Delta_{\varepsilon}+1\right)^{1 / 2} T_{\varepsilon}^{\theta} R_{\varepsilon}^{\theta} \| \\
& \leq \mathrm{e}^{-\operatorname{Re} \theta / 2} \mathcal{O}\left(\varepsilon^{1 / 2}\right) C^{\theta}
\end{aligned}
$$

where we employed the fact that $T_{\varepsilon}^{\theta} J=J T_{0}^{\theta}$ and Lemma 9.

Now we are in position to state our first convergence result of this section: 
Theorem 24. Assume that the metric graph $X_{0}$ is finite and non-compact, cf. (5.1). Then the complex dilated Laplacians $H_{0}^{\theta}$ and $H_{\varepsilon}^{\theta}$ are $\mathcal{O}\left(\varepsilon^{1 / 2}\right)$-close, i.e.

$$
\left\|J H_{0}^{\theta}-H_{\varepsilon}^{\theta} J\right\|_{2, \theta \rightarrow-2, \theta}=\left\|R_{\varepsilon}^{\theta} J-J R_{0}^{\theta}\right\|=\mathcal{O}\left(\varepsilon^{1 / 2}\right),
$$

where $\mathcal{O}\left(\varepsilon^{1 / 2}\right)$ depends on $\theta$.

Proof. The proof is essentially the same as for the non-dilated case. First we define operators on the scales of order one, specifically

$$
J^{1, \theta}: \mathcal{H}_{0}^{1, \theta} \longrightarrow \mathcal{H}_{\varepsilon}^{1, \theta} \quad \text { and } \quad J^{1, \theta \prime}: \mathcal{H}_{\varepsilon}^{1, \theta} \longrightarrow \mathcal{H}_{0}^{1, \theta}
$$

in exactly the same way as in (3.4) and (3.5). Recall that we do not consider the boundary points between the internal and external parts as vertices, i.e. the graph-like manifold does not have a vertex neighborhood there. Hence

$$
T_{\varepsilon}^{\theta} J^{1, \theta}=J^{1} T_{0}^{\theta} \quad \text { and } \quad T_{0}^{\theta} J^{1, \theta \prime}=J^{1 \prime} T_{\varepsilon}^{\theta},
$$

and then we have

$$
R_{\varepsilon}^{\theta} J-J R_{0}^{\theta}=R_{\varepsilon}^{\theta}\left[\left(J-J^{-1, \theta}\right) H_{0}^{\theta}+\left(J^{-1, \theta} H_{0}^{\theta}-H_{\varepsilon}^{\theta} J^{1, \theta}\right)+H_{\varepsilon}^{\theta}\left(J^{1, \theta}-J\right)\right] R_{0}^{\theta}
$$

where $J^{-1, \theta}:=\left(J^{1, \bar{\theta}}\right)^{*}$. The last difference at the right-hand side can be estimated by

$$
\begin{aligned}
\left\|R_{\varepsilon}^{\theta} H_{\varepsilon}^{\theta}\left(J^{1, \theta}-J\right) R_{0}^{\theta}\right\| \leq\left\|\mathrm{id}_{\varepsilon}-R_{\varepsilon}^{\theta}\right\| \cdot\left\|\left(J^{1, \theta}-J\right) R_{0}^{\theta}\right\| & \\
\leq\left(1+\left\|R_{\varepsilon}^{\theta}\right\|\right)\left\|T_{\varepsilon}^{-\theta}\right\| \cdot\left\|\left(J^{1}-J\right)\left(\Delta_{0}+1\right)^{-1 / 2}\right\|\left\|\left(\Delta_{0}+1\right)^{1 / 2} T_{0}^{\theta} R_{0}^{\theta}\right\| & \leq\left(1+C^{\theta}\right) \mathrm{e}^{-\operatorname{Re} \theta / 2} \mathcal{O}\left(\varepsilon^{1 / 2}\right) C^{\theta}
\end{aligned}
$$

using Lemma 14 and Lemma 23, and the first one can be treated similarly. For the remaining term we observe that in order to prove

$$
\left\|R_{\varepsilon}^{\theta}\left(J^{-1, \theta} H_{0}^{\theta}-H_{\varepsilon}^{\theta} J^{1, \theta}\right) R_{0}^{\theta}\right\|=\left\|J^{-1, \theta} H_{0}^{\theta}-H_{\varepsilon}^{\theta} J^{1, \theta}\right\|_{2, \theta \rightarrow-2, \theta} \leq \delta
$$

it suffices to show

$$
\left|\left\langle H_{0}^{\bar{\theta}} f, J^{1, \theta \prime} u\right\rangle-\left\langle J^{1, \theta} f, H_{\varepsilon}^{\theta} u\right\rangle\right| \leq \widetilde{\delta}\left\|T_{0}^{\bar{\theta}} f\right\|_{1}\left\|T_{\varepsilon}^{\theta} u\right\|_{1}
$$

for $f \in \mathcal{H}_{0}^{2, \bar{\theta}}$ and $u \in \mathcal{H}_{\varepsilon}^{2, \theta}$. From the compatibility, we obtain $\left\|T_{\varepsilon}^{\theta} u\right\|_{1} \leq C^{\theta}\|u\|_{2, \theta}$ and similarly for $f$; in particular, we can choose $\delta=\left(C^{\theta}\right)^{2} \widetilde{\delta}$, however, the estimate (5.4) is almost the same as in the non-dilated case given in Lemma 15.

As in the non-dilated case, on can develop a functional calculus for the pairs of operators $H_{0}^{\theta}$ and $H_{\varepsilon}^{\theta}-$ cf. [9, App. B]. Since now the operators are not self-ajoint, we only have a holomorphic functional calculus. In particular, we can show

$$
\left\|\mathbb{1}_{D}\left(H_{\varepsilon}^{\theta}\right) J-J \mathbb{1}_{D}\left(H_{0}^{\theta}\right)\right\| \leq \mathcal{O}\left(\varepsilon^{1 / 2}\right) \quad \text { and } \quad\left\|\mathbb{1}_{D}\left(H_{\varepsilon}^{\theta}\right)-J \mathbb{1}_{D}\left(H_{0}^{\theta}\right) J^{*}\right\| \leq \mathcal{O}\left(\varepsilon^{1 / 2}\right)
$$

for the spectral projections, provided $D$ is an open disc containing a single discrete eigenvalue $\lambda(0)$ of $H_{0}^{\theta}$. From here our main result on resonances follows:

Theorem 25. Assume that the metric graph $X_{0}$ is finite and non-compact, cf. (5.1). If $\lambda(0)$ is a resonance of the Laplacian $\Delta_{X_{0}}$ with a multiplicity $m>0$, then for a sufficiently small $\varepsilon>0$ there exist $m$ resonances $\lambda_{1}(\varepsilon), \ldots, \lambda_{m}(\varepsilon)$ of $\Delta_{X_{\varepsilon}}$, satisfying $\operatorname{Im} \lambda_{j}(\varepsilon)<0$ and not necessarily mutually different, which all converge to $\lambda(0)$ as $\varepsilon \rightarrow 0$. The same is true in the case when $\lambda(0)$ is an embedded eigenvalue of $\Delta_{X_{0}}$, except that then only $\operatorname{Im} \lambda_{j}(\varepsilon) \leq 0$ holds in general.

Finally, if the multiplicity of $\lambda(0)$ is one with a normalized eigenfunction $u^{\theta}(0)$ (corresponding to a resonance or embedded eigenvalue for $\Delta_{X_{0}}$ ), then there exists a normalized eigenfunction $u^{\theta}(\varepsilon)$ (related to the respective entity for $\Delta_{X_{\varepsilon}}$ ) on the graph-like manifold) such that

$$
\left\|J u^{\theta}(0)-u^{\theta}(\varepsilon)\right\|=\mathcal{O}\left(\varepsilon^{1 / 2}\right) .
$$




\section{ACKNOWLEDGMENTS}

The first author acknowledges a partial support by GAAS and MEYS of the Czech Republic under projects A100480501 and LC06002, the second one by DFG under the grant Po-1034/1-1.

\section{REFERENCES}

[1] J. Aguilar and J. M. Combes, A class of analytic perturbations for one-body Schrödinger Hamiltonians, Comm. Math. Phys. 22 (1971), 269-279.

[2] S. Albeverio, C. Cacciapuoti, and D. Finco, Coupling in the singular limit of thin quantum waveguides, J. Math. Phys. 48 (2007), 032103.

[3] E. Balslev and J. M. Combes, Spectral properties of many-body Schrödinger operators with dilatation-analytic interactions, Comm. Math. Phys. 22 (1971), 280-294.

[4] G. Berkolaiko, R. Carlson, St. A. Fulling, and P. Kuchment (eds.), Quantum graphs and their applications, Contemporary Mathematics, vol. 415, Providence, RI, American Mathematical Society, 2006.

[5] J.-M. Combes, P. Duclos, M. Klein, and R. Seiler, The shape resonance, Comm. Math. Phys. 110 (1987), 215-236.

[6] P. Exner, Open quantum systems and Feynman integrals, Fundamental Theories of Physics, D. Reidel, Dordrecht, 1985.

[7] P. Exner and C. Cacciapuoti, Nontrivial edge coupling from a Dirichlet network squeezing: the case of a bent waveguide, J. Phys. A 40 (2007), L511-L523.

[8] P. Exner and O. Post, Convergence of spectra of graph-like thin manifolds, Journal of Geometry and Physics 54 (2005), 77-115.

[9] _ Convergence of resonances on thin branched quantum wave guides, J. Math. Phys. 48 (2007), 092104.

[10] P. Exner and P. Šeba, Bound states in curved quantum waveguides, J. Math. Phys. 30 (1989), 2574-2580.

[11] M. I. Freidlin and A. D. Wentzell, Diffusion processes on graphs and the averaging principle, Ann. Probab. 21 (1993), 2215-2245.

[12] D. Grieser, Spectra of graph neighborhoods and scattering, arXiv:0710.3405v1 [math.SP]

[13] V. Kostrykin and R. Schrader, Kirchhoff's rule for quantum wires, J. Phys. A 32 (1999), 595-630.

[14] P. Kuchment, Quantum graphs: I. Some basic structures, Waves Random Media 14 (2004), S107-S128.

[15] P. Kuchment and H. Zeng, Convergence of spectra of mesoscopic systems collapsing onto a graph, J. Math. Anal. Appl. 258 (2001), 671-700.

[16] _ Asymptotics of spectra of Neumann Laplacians in thin domains, Advances in differential equations and mathematical physics (Birmingham, AL, 2002), Contemp. Math., vol. 327, Amer. Math. Soc., Providence, RI, 2003, pp. 199-213.

[17] S. Molchanov and B. Vainberg, Scattering solutions in a network of thin fibers: small diameter asymptotics, Commun. Math. Phys. 273 (2007), 533-559.

[18] O. Post, Branched quantum wave guides with Dirichlet boundary conditions: the decoupling case, Journal of Physics A: Mathematical and General 38 (2005), 4917-4931.

[19] _ Spectral convergence of quasi-one-dimensional spaces, Ann. Henri Poincaré 7 (2006), $933-973$.

[20] J. Rubinstein and M. Schatzman, Variational problems on multiply connected thin strips. I. Basic estimates and convergence of the Laplacian spectrum, Arch. Ration. Mech. Anal. 160 (2001), 271-308.

[21] K. Ruedenberg and C. W. Scherr, Free-electron network model for conjugated systems, I. Theory, J. Chem. Phys. 21 (1953), 1565-1581.

Department of Theoretical Physics, NPI, Academy of Sciences, 25068 Řež Near Prague, and

Doppler Institute, Czech Technical University, Břehová 7, 11519 Prague, Czechia

E-mail address: exner@ujf.cas.cz

Institut für Mathematik, Humboldt-Universität, Rudower Chaussee 25, 12489 Berlin, Germany E-mail address: post@math.hu-berlin.de 